\title{
Are proximal colorectal cancers always associated with distal adenomas?
}

\section{A J M Watson}

Only half of proximal colon cancers are associated with adenomas in the distal colon. This has important implications for the selection of the initial investigation for colorectal cancer screening

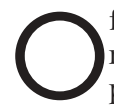

all the common cancers, colorectal cancer is the best suited to prevention through screening as it is derived from benign adenomas which can be easily detected and removed. The best screening investigation remains much debated. Many argue that colonoscopy is superior to other techniques because it has the highest sensitivity ( $>90 \%$ ) and examines the whole colon. ${ }^{1}$ However, it has a number of important disadvantages. Firstly, it is potentially dangerous. Perforation rates of 1 in 1000-1 in 20000 have been found in large studies from the USA and Germany. ${ }^{2}$ Colonoscopy is also expensive and requires highly skilled operators who are in short supply. ${ }^{4}$ For these reasons investigators have sought a screening strategy that reduces the number of colonoscopies undertaken. A study from St Mark's Hospital of the long term risk of colorectal cancer in patients with rectosigmoid adenomas found that $88 \%$ of cancers developed in patients with high risk (villous, tubulovillus histology, or $>10 \mathrm{~mm}$ in diameter) rectosigmoid adenomas. ${ }^{5}$ This study led Atkin et al to propose that a single examination with a flexible sigmoidoscopy leading to full colonoscopy in patients with high risk rectosigmoid adenomas would be a cost effective and safe screening protocol. ${ }^{6}$ This strategy is now being tested in a randomised controlled clinical trial. Baseline findings have already established the perforation rate of diagnostic flexible sigmoidoscopy to be considerably lower than that of colonoscopy at 1 in 40000 .

The crucial assumption for the use of flexible sigmoidoscopy as the initial screening test is that all proximal cancers are associated with distal adenomas. If this is not true then the findings at flexible sigmoidoscopy will not trigger the colonoscopy required to make the diagnosis of proximal colorectal cancer. This assumption is brought into sharper focus by the increasing proportion of colorectal cancers arising in the right colon. ${ }^{8}$ In this issue of Gut, Gondal and colleagues ${ }^{9}$ investigated the association of distal adenomas with proximal colorectal neoplasia [see page 398]. The investigators took advantage of the Norwegian Colorectal Cancer Prevention study (NORCCAP) in which 20780 individuals aged 54-64 years, selected randomly from the population registry of Oslo and Telemark County, were offered a once only examination by flexible sigmoidoscopy or a combination of flexible sigmoidoscopy and faecal occult blood testing. Individuals diagnosed as having an adenoma of any size were offered full colonoscopy. The current study examined the risk of proximal adenomas and carcinomas in the 2154 individuals (17\% of the total screenees) who were found to have distal neoplasms. Of these, 1833 individuals were studied. Twenty one per cent of subjects had colonic neoplasms proximal to the level reached by flexible sigmoidoscopy and a further $5 \%$ of subjects had proximal advanced neoplasms (PAN) defined as high risk adenomas or carcinomas. The risk of PAN increased threefold in subjects with distal adenoma $>10 \mathrm{~mm}$ in diameter or containing villous components. The investigators then calculated the number of PAN that would have been missed depending on the threshold criteria for offering colonoscopy. If the threshold criteria for colonoscopy had been more than one adenoma or a single high risk adenoma (as defined by a diameter $>10 \mathrm{~mm}$ or villous components or showing severe dysplasia) then $38 \%$ of PAN would have been missed, including $17 \%$ of proximal carcinomas. Furthermore, the tendency to miss PAN was found to increase with the age of the subject. On the other hand, implementation of these strict threshold criteria would have resulted in $66 \%$ fewer colonoscopies being undertaken.

A particularly interesting feature of the study was that colonoscopes were used to perform many of the flexible sigmoidoscopic examinations with the extent of examination limited by the degree of bowel cleansing from a single sorbitol enema. In this way the investigators were able to examine a greater proportion of the colon than is usually possible with a conventional flexible sigmoidoscope. This additional examination above that of conventional flexible sigmoidoscopy resulted in a further 3\% of patients being offered full colonoscopy and three proximal carcinomas being detected in subjects who would have otherwise been misclassified as having no neoplasia. The authors conclude that the finding of any adenoma at flexible sigmoidoscopy should trigger a full colonoscopy. They recommend that the initial examination should be an unsedated examination with a colonoscope after a simple enema.

This study is consistent with previous findings that $46-52 \%$ of PAN are not accompanied by distal polyps. ${ }^{10} 11$ Addition of faecal occult blood testing to flexible sigmoidoscopy does not significantly increase the detection of advanced neoplasia. ${ }^{12}$ One is therefore left with the conclusion that colonoscopy remains the most sensitive screening tool and, if performed by a skilled operator, is reasonably safe. No screening technique will entirely eliminate the risk of colorectal cancer. Risk reduction is all that can be achieved and this must be carefully explained to patients. Flexible sigmoidoscopy is safer, cheaper, and more convenient for patients than colonoscopy but at the cost of lower efficacy for preventing and detecting cancer.

\section{Gut 2003;52:317-318}

\section{Author's affiliation}

A J M Watson, Department of Medicine, University of Liverpool, Daulby St, Liverpool L69 3GA, UK; alastair.watson@liv.ac.uk

\section{REFERENCES}

Ransohoff DF, Sandler RS. Clinical practice. Screening for colorectal cancer. N Engl J Med 2002;346:40-4

2 Tran DQ, Rosen L, Kim R, et al. Actual colonoscopy: what are the risks of perforation? Am Surg 2001;67:845-7.

3 Sieg A, Hachmoeller-Eisenbach U, Eisenbach T. Prospective evaluation of complications in outpatient $\mathrm{Gl}$ endoscopy: a survey among German gastroenterologists. Gastrointest Endosc 2001;53:620-7.

4 Detsky AS. Screening for colon cancer-can we afford colonoscopy? N Engl J Med 2001:345:607-8.

5 Atkin WS, Morson BC, Cuzick J. Long-term risk of colorectal cancer after excision of rectosigmoid adenomas. N Engl J Med 1992;326:658-62.

6 Atkin WS, Cuzick J, Northover JM, et al. Prevention of colorectal cancer by once- only sigmoidoscopy. Lancet 1993;341:736-40.

7 Single flexible sigmoidoscopy screening to prevent colorectal cancer: baseline findings of a UK multicentre randomised trial. Lancet 2002;359:1291-300.

8 McCallion K, Mitchell RM, Wilson RH, et al. Flexible sigmoidoscopy and the changing distribution of colorectal cancer: implications distribution of colorectal cancer: implico

9 Gondal G, Grotmal T, Hofstad B, et al. Grading of distal colorectal adenomas as predictors for proximal colonic neoplasia and choice of endoscope in population screening: experience from the Norwegian Colorectal Cancer Prevention study (NORCCAP). Gut Cancer Prevention s 2003;52:398-403. 
10 Imperiale TF, Wagner DR, Lin CY, et al. Risk of advanced proximal neoplasms in asymptomatic adults according to the distal colorectal findings. N Engl J Med 2000;343: 169-74.
11 Lieberman DA, Weiss DG, Bond JH, et al. Use of colonoscopy to screen asymptomatic adults for colorectal cancer. Veterans Affairs Cooperative Study Group 380. N Engl J Med 2000;343: 162-8.
12 Lieberman DA, Weiss DG. One-time screening for colorectal cancer with combined fecal occult-blood testing and examination of the distal colon. N EnglJ Med 2001;345:555-60.

\section{Lessons from liver transplantation: flip, flop, and why?}

\section{P C Adams}

\section{A case of hereditary haemochromatosis developing in a non-hereditary haemochromatosis recipient following transplantation of a C282Y heterozygous donor liver}

$\mathrm{T}$

he widespread application of liver trans plantation has been the single mos important therapy to extend long term survival in patients with a variety of acute and chronic liver diseases. A fringe benefit of liver transplantation has been identification, confirmation, and cure of the metabolic basis of diseases. ${ }^{1}$ Haemophilia patients transplanted for chronic hepatitis $\mathrm{C}$ are cured of their clotting disorder, and patients transplanted for Wilson disease show no signs of copper accumulation following transplantation. Although many transplant recipients have mild to moderate iron overload at the time of transplantation, liver transplantation has been uncommon in homozygotes for the C282Y mutation of the HFE gene for haemochromatosis, and the metabolic effects on iron metabolism have not been clearly established. ${ }^{2}$ Studies in which C282Y homozygous donors have been transplanted into normal recipients have usually demonstrated a return to normal liver iron concentrations over time, and transplantation of a $\mathrm{C} 282 \mathrm{Y}$ homozygous liver and intestine into a normal recipient has led to iron accumulation necessitating venesection therapy. ${ }^{3}$ These studies have suggested that the intestine is the major organ involved in the pathogenesis of haemochromatosis although systemic abnormalities in macrophage and lymphocyte function have been described. A major unresolved issue in haemochromatosis is why some C282Y homozygotes have cirrhosis and other organ damage while as many as $50 \%$ of $\mathrm{C} 282 \mathrm{Y}$ homozygotes will have no biochemical abnormalities of transferrin saturation or ferritin. ${ }^{4}$

In this issue of Gut, Wigg and colleagues $^{5}$ describe a fascinating case of iron accumulation following liver transplantation [see page 433]. The 44 year old female donor was a C282Y heterozygote or carrier with a slight elevation in hepatic iron concentration. The recipient had mild iron overload secondary to alcoholic siderosis and was found to have a new polymorphism in the HFE gene (R6S). Four years later the recipient was found to have severe hepatic siderosis.

The tantalising aspect of this unusual case report is the suggestion that two independent mutations could be synergistic to result in iron overload. This concept has been studied in haemochromatosis as several investigators have searched for concomitant mutations in non- HFE iron transport genes such as DMTl and transferrin receptor genes. ${ }^{6}$ The hypothesis could be that the $\mathrm{C} 282 \mathrm{Y}$ homozygote without any other coexisting mutations would be unaffected. In the presence of another common mutation ( since $>50 \%$ of $\mathrm{C} 282 \mathrm{Y}$ homozygotes have iron abnormalities), iron accumulation would occur and would lead to organ dysfunction. This "two hit" hypothesis has been proposed in other diseases such as $\alpha_{1}$ - antitrypsin deficiency and non-alcoholic steatohepatitis. However, if expression of haemochromatosis is dependent on co-modifying genes, pedigrees of non-expressing cases would be more common than currently reported. As females are significantly less affected than men, and alcohol worsens the disease severity in haemochromatosis, expression of haemochromatosis could also be an example of a gene-environmental interaction.

There are several unexplained aspects to the current report. A simple C282Y heterozygote would presumably express this defect in the liver and intestine and yet most C282Y heterozygotes have normal iron studies, and significant iron overload in simple heterozygotes is extremely rare. Transplantation using $\mathrm{C} 282 \mathrm{Y}$ heterozygous donors and recipients has been reported. ${ }^{7}$ At our centre, we have transplanted three $\mathrm{C} 282 \mathrm{Y}$ heterozygous livers into three $\mathrm{C} 282 \mathrm{Y}$ heterozygous recipients without the apparent development of iron overload.
The presence of a base pair change (R6S) is not synonymous with a major metabolic effect on iron metabolism. Further studies will be required on this polymorphism to determine if functional changes occur in the HFE protein, and it would be uncommon for heterozygotes of any HFE mutation to have significant changes in iron metabolism. For example, the H63D mutation does not lead to iron overload in H63D heterozygotes.

To further complicate the clinical picture, recently a ferroportin mutation has been described leading to moderate to severe iron overload in patients. Many of the patients in the original report had a normal transferrin saturation and ferritin. ${ }^{8}$ If iron tests cannot predict the development of iron overload, there may be cases of occult iron overload in the general population or post liver transplant that have not come to medical attention.

The unusual case described in this report may never occur again but this and other cases resulting from inadvertent experiments in transplantation will continue to stimulate ideas and insights into the mysteries of iron metabolism.

Gut 2003;52:317-318

\section{Author's affiliation}

P C Adams, Department of Medicine, London Health Sciences Centre, 339 Windermere Rd, London, Ontario, Canada N6A 5A5;

padams@uwo.ca

\section{REFERENCES}

1 Adams PC. A quarter century of live transplantation-learning from livers. Can J Gastroenterol 1989;3: 162-4.

2 Powell LW. Does transplantation of the liver cure genetic hemochromatosis? J Hepatol 1992:16:259-61.

3 Adams PC, Alanen K, Preshaw R, et al. Transplantation of haemochromatosis live and intestine into a normal recipient. Gut 1999;45:783

4 Adams PC. Non-expressing C282Y homozygotes for hemochromatosis: minority or majority of cases? Mol Genet Metab 2000:71:81-6

5 Wigg AJ, Harley H, Casey G. Heterozygous recipient and donor HFE mutations associated with a hereditary haemochromatosis phenotype after liver transplantation. Gut 2003; 52:433-5.

6 Sachot S, Moirand R, Jouanolle AM, et al. Low penetrant hemochromatosis phenotype in eight families: no evidence of modifiers in the MHC region. Blood Cells Mol Dis 2001;27:518-29.

7 Alanen K, Chakrabarti S, Rawlins J, et al. Prevalence of the C282Y mutation of the hemochromatosis gene in liver transplant recipients and donors. Hepatology recipients and donors

8 Niajou O, Vaessen N, Joosse M, et al. A mutation in $\mathrm{SLC} 11 \mathrm{~A} 3$ is associated with autosomal dominant hemochromatosis. Nat Genet 2001;28:214-15. 